\section{On the Equivalence of Electromagnetic and Clock-Transport Synchronization in Noninertial Frames and Gravitational Fields}

Helmut Rumpf

Institut für Theoretische Physik, Universität Wien, Österreich

\section{Z. Naturforsch. 40 a, 92-93 (1985);} received September 14, 1984

Synchronization by slow clock transport is shown to be equivalent to that by electromagnetic signals for clocks moving along the trajectories of a timelike Killing vector field, provided the gravitational redshift is corrected for and the synchronization paths are the same.

Clock synchronization plays an important role in many gedanken experiments of special relativity. Usually one considers clocks resting in one and the same inertial system. Synchronization of two clocks is achieved either (i) by demanding that a light flash emitted from the midpoint between the two clocks should reach the clocks at instants corresponding to identical clock readings ("Einstein synchronization") [1] or (ii) by slowly moving a third clock from one clock to the other and requiring that the readings of the third clock and one of the other clocks should be the same at departure and arrival, respectively. It can easily be shown that (i) and (ii) are equivalent in the limit as the velocity of the synchronizing clock used in clock-transport synchronization goes to zero.

In this note we address the question whether electromagnetic and clock-transport synchronization (in the zerovelocity limit) remain equivalent under more general circumstances, namely if the clocks that are to be synchronized move along non-inertial trajectories in Minkowski space or are influenced by a gravitational field. We shall show by an elementary proof that the above equivalence holds in an arbitrary stationary metric, provided (i) the clocks that are to be synchronized follow trajectories of a timelike Killing vector field, (ii) proper time is replaced by the Killing parameter (i.e. the (pseudo-)gravitational redshift is corrected for in the clock readings) and (iii) the synchronization paths (of the light ray and the synchronizing clock) are the same. Assumption (iii) is necessary because synchronization is path-dependent, if the Killing vector field is not hypersurface-orthogonal. In the case of Einstein synchronization this fact is well-known from the example of the rotating disk [2]. A synchronization path can be specified by a curve on a spacelike slice or, more conveniently, by the timelike two-dimensional surface defined by that curve and the Killing trajectories. We shall refer in the following to this two-dimensional surface as

Reprint requests to Dr. H. Rumpf, Institut für Theoretische Physik, Universität Wien, Boltzmanngasse 5, A-1090 Wien, Österreich. the "synchronization sheet". According to assumption (iii) both the null lines corresponding to the synchronizing light rays (which may be curved by mirrors etc. and hence need not coincide with null geodesics) and the worldline of the synchronizing clock have to lie within the synchronization sheet. Without loss of generality the synchronization path, and hence the surface, may be taken as non-selfintersecting, since one can always decompose a path into nonintersecting pieces and synchronization along a given path will turn out to be transitive.

In order to prove the above assertion we consider the metric induced on the two-dimensional synchronization sheet by the full spacetime metric. It is again stationary; hence there exists a coordinate system in which it takes the form

$$
\mathrm{d} s_{(2)}^{2}=f_{00}(x) \mathrm{d} t^{2}+2 f_{01}(x) \mathrm{d} t \mathrm{~d} x+f_{11}(x) \mathrm{d} x^{2}
$$

and in which the curves $x=$ const are Killing trajectories. But every two-dimensional stationary metric is also static (or, in geometrical terms, there exist curves orthogonal to the Killing vector field, whose projections on a given three-dimensional spacelike slice may be specified arbitrarily; these curves will turn out to be just the "curves of simultaneity" defined by the synchronization process). This is made explicit by the coordinate transformation

$$
\begin{aligned}
\tau & =t+\int^{x}\left(f_{01} / f_{00}\right) \mathrm{d} x^{\prime} \\
\xi & =\int^{x}\left(-f_{11}+f_{01}^{2} / f_{00}\right)^{1 / 2} \mathrm{~d} x^{\prime}
\end{aligned}
$$

upon which (1) becomes

$$
\mathrm{d} s_{(2)}^{2}=f(\xi) \mathrm{d} \tau^{2}-\mathrm{d} \xi^{2}
$$

and the Killing trajectories are given by $\xi=$ const.

To complete the proof it will be convenient to use the following equivalent definition of the Einstein synchronization of two clocks: Consider the future and past directed null lines emanating from a point of the worldline of one clock that lie within the synchronization sheet. They intersect the worldline of the other clock at two points. The middle between these two points defines the instant at which the other clock should show the same reading as the first clock at the instant of emanation of the null lines.

Now the synchronizing light rays are indeed null geodesics with respect to the induced metric (4). Thus we are led to consider the "light cone" (within the synchronization sheet) belonging to a point with, say, $\tau=0$. By time inversion symmetry (which is a consequence of staticity), this light cone will intersect any Killing line at two instants, $\tau=T$ and $\tau=-T$. Using now the reformulated definition of Einstein synchronization given previously, we conclude that the spacelike curves $\tau=$ const are "curves of simultaneity" with respect to the electromagnetic synchronization. But it is also obvious that a clock which moves on a worldline with four-velocity almost parallel to the Killing vector field will measure the redshift-corrected propertime interval $\int \mathrm{d} s f^{-1 / 2}$ between encountering two different Killing curves to be only slightly shorter than the difference $\Delta \tau$ of the coordinate times $\tau$ corresponding to these 
encounters. The discrepancy can be made arbitrarily small (though $\Delta \tau$ will grow indefinitely). This establishes the equivalence of clock-transport and Einstein synchronization under the provisions made above.

The above identification of the "curves of simultaneity" implies immediately that a closely spaced band of clocks will always give a synchronization equivalent to that with infinitely spaced clocks, if the path is not changed. This explains in general an observation made recently [3] in the special case of the rotating disk. Furthermore it is obvious from the above proof that the conditions under which Einstein and clock-transport synchronization are equivalent can be generalized in the following way: Instead of assuming that the space-time metric is stationary it suffices to assume that a given synchronization path may be imbedded in a two-dimensional timelike sheet whose induced metric is stationary and that the clock worldlines are trajectories of a timelike Killing vector field with respect to the induced metric.

In order to demonstrate the simplifying power of the geometrical concepts used in the equivalence proof we conclude this article by a consideration of the rotating disk, which has not always received the most concise treatment in the literature [4]. The relevant Killing vector field has components $\left(1,0, c^{-1} \omega r\right)$ with respect to the orthonormal triad $\left\{\frac{\partial}{\partial t}, \frac{\partial}{\partial r}, \frac{1}{r} \frac{\partial}{\partial \varphi}\right\}$, where $r$ and $\varphi$ are cylinder coordinates and $\omega$ is the angular velocity of the disk. We are interested in a circular synchronization path whose center coincides with that of the disk and whose radius is $r$. The corresponding "curve of simultaneity" orthogonal to the Killing vector field has tangent vectors $\left(1,0, c \omega^{-1} r^{-1}\right)$ and may be visualized as a helical curve in a space-time diagram (this, of course, is also true for the Killing trajectories themselves). The "pitch" ("Ganghöhe") of the helix is given by

$$
\begin{aligned}
c^{-1} \times \text { circumference } \times \text { slope } & =c^{-1} \cdot 2 \pi r \cdot c^{-1 \bullet} \omega r \\
& =2 \pi c^{-2} r^{2} \omega .
\end{aligned}
$$

Taking into account the flat geometry of the cylindrical synchronization sheet we obtain for the "desynchronization" (difference in proper time of a Killing trajectory between two successive intersections with the curve of simultaneity)

$$
\Delta=\left(1-v^{2} / c^{2}\right)^{-1 / 2} 2 \pi r^{2} \omega / c^{2},
$$

[1] A. Einstein, The Meaning of Relativity, 5th edition, Princeton University Press, Princeton, N.J. 1972.

[2] L. D. Landau and E. M. Lifshitz, The Classical Theory of Fields, 8th German edition, Akademie-Verlag, Berlin 1976.

[3] J. M. Cohen, H. E. Moses, and A. Rosenblum, Phys. Rev. Lett. 51, 1501 (1983). where $v=r \omega$. On the other hand a direct calculation of the desynchronization due to slow clock transport around the closed synchronization path considered yields

$$
\begin{aligned}
\Delta & =\int_{t_{a}}^{t_{b}}\left(\sqrt{1-v^{2} / c^{2}}-\sqrt{\left.1-v^{\prime 2 / c^{2}}\right)} \mathrm{d} t^{\prime}\right. \\
& \cong \sqrt{1-v^{2} / c^{2}} \int_{t_{a}}^{t_{b}} \frac{1}{2} \frac{v^{\prime 2}-v^{2}}{c^{2}-v^{2}} \mathrm{~d} t^{\prime} \\
& \cong\left(1-v^{2} / c^{2}\right)^{-1 / 2} 2 \pi r^{2} \omega / c^{2}
\end{aligned}
$$

i.e. the same result. Here $v^{\prime}$ is the velocity of the synchronizing clock (assumed to be only slightly different from $v$ ) and $t_{b}-t_{a}$ is the time for this clock to make one revolution relative to the disk. Now $2 \pi r\left(1-v^{2} / c^{2}\right)^{-1 / 2}$ is the metric length of the curve of simultaneity, and $\pi r^{2}\left(1-v^{2} / c^{2}\right)^{-1 / 2}$ is the area enclosed by the synchronization circle as measured in the corotating frame. It is not difficult to show that for an arbitrary closed synchronization path

$$
\Delta=2\left(\omega / c^{2}\right) A,
$$

where $A$ is the area on the disk (measured in the corotating system) enclosed by the synchronization path.

Formulas (5) and (6) describe also the genuine desynchronization of a closed chain of clocks on the earth's equator or in identical circular orbit around the earth, if the clocks display $\left(\mathrm{d} t^{2}-r^{2} \mathrm{~d} \varphi^{2}\right)^{1 / 2} \cong g_{00}^{-1 / 2} \mathrm{~d} s$ instead of the proper time interval $\mathrm{d} s$, where $t$ is the Schwarzschild time coordinate and $g_{00}=1-2 G M / r c^{2}$ is a component of the Schwarzschild metric ( $M$ is the mass of the earth). The reason is that the Schwarzschild metric describes to a good approximation the gravitational field in the vicinity of the earth (neglecting the gravitational effects of the other celestial bodies and of the earth's rotation). Note that the earth is rotating in the Schwarzschild frame and that due to the small velocities (as compared with $c$ ) involved the red-shift corrected proper time is practically identical with the Schwarzschild coordinate time $t$. Under the provision just made one can also generalize (7) to the case of an arbitrary closed synchronization path on the surface of the earth. For this $A$ simply has to be taken as the area enclosed by the projection of the synchronization path on the equatorial plane. For an up-to-date review of the experimental aspects of clock synchronization in the vicinity of the earth we refer the reader to [5] and the references cited therein.

It is a pleasure to acknowledge an interesting discussion with Professor S. Bazański.

[4] J. M. Cohen and H. E. Moses, Phys. Rev. Lett. 39, 1641 (1977); D. B. Lichtenberg and R. G. Newton, Phys. Rev. D 19, 1268 (1979); F. Curtis Michel, Phys. Rev. D 19, 1271 (1979); J. M. Cohen, C. Ftaclas, and H. Moses, Phys. Rev. D 19, 1273 (1979).

[5] N. Ashby and D. W. Allan, Phys. Rev. Lett. 53, 1858 (1984). 


\section{Elementary Derivation of the Dirac Equation. VIII}

Hans Sallhofer

Z. Naturforsch. 40 a, 94-95 (1985);

received November 28,1984

The momentum balance of Dirac-like electrodynamics is established in Da Silveira's notation.

The notation of De Silveira [1] obviously is of great heuristic importance. In establishing the Maxwell-Dirac isomorphy, for instance, it avoids the artifice [2, (6)] and, in addition, brings about the Darwin relation [2, (7)] automatically. Therefore we want to investigate how the momentum balance [4, (11)] is represented in Da Silveira's formalism.

According to De Silveira we may put the Diraclike electrodynamics $[2,(5)]$ into the form

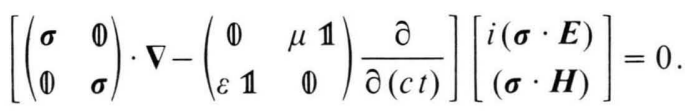

Multiplication by the matrix

$$
\left(\begin{array}{ll}
0 & \mathbb{1} \\
\mathbb{1} & 0
\end{array}\right)
$$

from the left yields

$\left[\left(\begin{array}{ll}\mathbb{0} & \boldsymbol{\sigma} \\ \boldsymbol{\sigma} & \mathbb{0}\end{array}\right) \cdot \nabla-\left(\begin{array}{cc}\varepsilon \mathbb{1} & \mathbb{0} \\ \mathbb{0} & \mu \mathbb{1}\end{array}\right) \frac{\partial}{\partial(c t)}\right]\left[\begin{array}{c}i(\boldsymbol{\sigma} \cdot \boldsymbol{E}) \\ (\boldsymbol{\sigma} \cdot \boldsymbol{H})\end{array}\right]=0$.

If we identify, as in [2, (7)] and [2, (8)],

$$
\begin{aligned}
\gamma^{(j)} & =\left(\begin{array}{ll}
\mathbb{0} & \boldsymbol{\sigma}_{j} \\
\boldsymbol{\sigma}_{j} & \mathbb{1}
\end{array}\right), \quad \gamma^{(k)}=-\left(\begin{array}{cc}
\varepsilon \mathbb{1} & \mathbb{0} \\
\mathbb{0} & \mu \mathbb{1}
\end{array}\right), \\
\Psi & =\left[\begin{array}{c}
i(\boldsymbol{\sigma} \cdot \boldsymbol{E}) \\
(\boldsymbol{\sigma} \cdot \boldsymbol{H})
\end{array}\right],
\end{aligned}
$$

then (3) is identical with $[2,(8)]$ :

$$
\left[\gamma \cdot \nabla+\gamma^{(k)} \partial_{k}\right] \Psi=0 .
$$

The wavefunction now is a matrix with four rows and two columns:

$$
\Psi=\left[\begin{array}{c}
i(\boldsymbol{\sigma} \cdot \boldsymbol{E}) \\
(\boldsymbol{\sigma} \cdot \boldsymbol{H})
\end{array}\right]=\begin{array}{cc}
i E_{3} & i\left(E_{1}-i E_{2}\right) \\
i\left(E_{1}+i E_{2}\right) & -i E_{3} \\
H_{3} & H_{1}-i H_{2} \\
H_{1}+i H_{2} & -H_{3}
\end{array}
$$

Reprint requests to Dr. Hans Sallhofer, Fischer-Strasse 12, A-5280 Braunau, Austria. where $[2,(7)]$ is represented by the first column.

In order to obtain the energy relations we repeat the procedure leading to $[3,(5)]$. For this we take (5) and its hermitian conjugate. They read explicitly

$$
\begin{aligned}
& \gamma \cdot(\nabla \Psi)+\frac{1}{c} \gamma_{k}\left(\frac{\partial}{\partial t} \Psi\right)=0, \\
& \left(\nabla \Psi^{k c}\right) \cdot \gamma+\frac{1}{c}\left(\frac{\partial}{\partial t} \Psi^{k c}\right) \gamma_{k}=0,
\end{aligned}
$$

where $\Psi^{k c}$ has the form

$$
\begin{aligned}
& \Psi^{k c}=\left[-i(\boldsymbol{\sigma} \cdot \boldsymbol{E}),\left(\boldsymbol{\sigma} \cdot \boldsymbol{H}^{*}\right)\right] \\
& =\left[\begin{array}{cccc}
-i E_{3}^{*} & -i\left(E_{1}^{*}-i E_{2}^{*}\right) & H_{3}^{*} & H_{1}^{*}-i H_{2}^{*} \\
-i\left(E_{1}^{*}+i E_{2}^{*}\right) & i E_{3}^{*} & H_{1}^{*}+i H_{2}^{*} & -H_{3}^{*}
\end{array}\right] .
\end{aligned}
$$

Multiplication of (7) by $\Psi^{k c}$ from the left, (8) by $\Psi$ from the right, and subsequent addition yields

$$
\frac{1}{c} \frac{\partial}{\partial t}\left(\Psi^{k c} \gamma_{k} \Psi\right)+\operatorname{div}\left(\Psi^{k c} \gamma \Psi\right)=0
$$

The two terms are

$$
\begin{aligned}
\Psi^{k c} \gamma_{4} \Psi & =-\left[-i\left(\boldsymbol{\sigma} \cdot \boldsymbol{E}^{*}\right),\left(\boldsymbol{\sigma} \cdot \boldsymbol{H}^{*}\right)\right]\left(\begin{array}{cc}
\varepsilon & 0 \\
0 & \mu
\end{array}\right)\left[\begin{array}{c}
i(\boldsymbol{\sigma} \cdot \boldsymbol{E}) \\
(\boldsymbol{\sigma} \cdot \boldsymbol{H})
\end{array}\right] \\
& =-\varepsilon\left(\boldsymbol{\sigma} \cdot \boldsymbol{E}^{*}\right)(\boldsymbol{\sigma} \cdot \boldsymbol{E})-\mu\left(\boldsymbol{\sigma} \cdot \boldsymbol{H}^{*}\right)(\boldsymbol{\sigma} \cdot \boldsymbol{H})(11)
\end{aligned}
$$

and

$$
\begin{aligned}
\Psi^{k c} \gamma \Psi & =\left[-i\left(\boldsymbol{\sigma} \cdot \boldsymbol{E}^{*}\right),\left(\boldsymbol{\sigma} \cdot \boldsymbol{H}^{*}\right)\right]\left(\begin{array}{ll}
0 & \boldsymbol{\sigma} \\
\boldsymbol{\sigma} & 0
\end{array}\right)\left[\begin{array}{c}
i(\boldsymbol{\sigma} \cdot \boldsymbol{E}) \\
(\boldsymbol{\sigma} \cdot \boldsymbol{H})
\end{array}\right] \\
= & i\left[\left(\boldsymbol{\sigma} \cdot \boldsymbol{H}^{*}\right) \boldsymbol{\sigma}(\boldsymbol{\sigma} \cdot \boldsymbol{E})-\left(\boldsymbol{\sigma} \cdot \boldsymbol{E}^{*}\right) \boldsymbol{\sigma}(\boldsymbol{\sigma} \cdot \boldsymbol{H})\right] .
\end{aligned}
$$

With the help of the two relations

$$
(\boldsymbol{\sigma} \cdot \boldsymbol{A})(\boldsymbol{\sigma} \cdot \boldsymbol{B})=(\boldsymbol{A} \cdot \boldsymbol{B})+i \boldsymbol{\sigma} \times(\boldsymbol{A} \times \boldsymbol{B})
$$

and

$(\sigma \cdot A) \sigma(\sigma \cdot B)$

$$
=\boldsymbol{A}(\boldsymbol{\sigma} \cdot \boldsymbol{B})+(\boldsymbol{\sigma} \cdot \boldsymbol{A}) \boldsymbol{B}-i(\boldsymbol{A} \times \boldsymbol{B})-(\boldsymbol{A} \cdot \boldsymbol{B}) \boldsymbol{\sigma},
$$

we get

$$
\begin{aligned}
\Psi^{k c} \gamma_{k} \Psi= & -\varepsilon\left[\left(\boldsymbol{E}^{\mathrm{Re}}\right)^{2}+\left(\boldsymbol{E}^{\mathrm{Im}}\right)^{2}\right]-\mu\left[\left(\boldsymbol{H}^{\mathrm{Re}}\right)^{2}+\left(\boldsymbol{H}^{\mathrm{Im}}\right)^{2}\right] \\
& -2 \varepsilon \boldsymbol{\sigma} \cdot\left(\boldsymbol{E}^{\mathrm{Im}} \times \boldsymbol{E}^{\mathrm{Re}}\right)-2 \mu \boldsymbol{\sigma} \cdot\left(\boldsymbol{H}^{\mathrm{Im}} \times \boldsymbol{H}^{\mathrm{Re}}\right)
\end{aligned}
$$

and

$$
\begin{aligned}
\Psi^{k c} \gamma \Psi= & -2\left[\left(\boldsymbol{E}^{\mathrm{Re}} \times \boldsymbol{H}^{\mathrm{Re}}\right)+\left(\boldsymbol{E}^{\mathrm{Im}} \times \boldsymbol{H}^{\mathrm{Im}}\right)\right. \\
& +\boldsymbol{H}^{\mathrm{Re}}\left(\boldsymbol{\sigma} \cdot \boldsymbol{E}^{\mathrm{Im}}\right)-\boldsymbol{H}^{\mathrm{Im}}\left(\boldsymbol{\sigma} \cdot \boldsymbol{E}^{\mathrm{Re}}\right) \\
& +\boldsymbol{E}^{\mathrm{Im}}\left(\boldsymbol{\sigma} \cdot \boldsymbol{H}^{\mathrm{Re}}\right)-\boldsymbol{E}^{\mathrm{Re}}\left(\boldsymbol{\sigma} \cdot \boldsymbol{H}^{\mathrm{Im}}\right) \\
& \left.+\left(\boldsymbol{E}^{\mathrm{Re}} \cdot \boldsymbol{H}^{\mathrm{Im}}\right) \boldsymbol{\sigma}-\left(\boldsymbol{E}^{\mathrm{Im}} \cdot \boldsymbol{H}^{\mathrm{Re}}\right) \boldsymbol{\sigma}\right] .
\end{aligned}
$$


Thus (10) becomes

$$
\begin{aligned}
& \left\{\frac{1}{c} \frac{\partial}{\partial t} \frac{1}{2}\left[\varepsilon\left(\boldsymbol{E}^{\mathrm{Re}}\right)^{2}+\mu\left(\boldsymbol{H}^{\mathrm{Re}}\right)^{2}\right]+\operatorname{div}\left(\boldsymbol{E}^{\mathrm{Re}} \times \boldsymbol{H}^{\mathrm{Re}}\right)\right\} \\
& +\left\{\frac{1}{c} \frac{\partial}{\partial t} \frac{1}{2}\left[\varepsilon\left(\boldsymbol{E}^{\mathrm{Im}}\right)^{2}+\mu\left(\boldsymbol{H}^{\mathrm{Im}}\right)^{2}\right]+\operatorname{div}\left(\boldsymbol{E}^{\mathrm{Im}} \times \boldsymbol{H}^{\mathrm{Im}}\right)\right\} \\
& +\frac{1}{c} \frac{\partial}{\partial t}\left[\varepsilon\left(\boldsymbol{E}^{\mathrm{Im}} \times \boldsymbol{E}^{\mathrm{Re}}\right)+\mu\left(\boldsymbol{H}^{\mathrm{Im}} \times \boldsymbol{H}^{\mathrm{Re}}\right)\right] \cdot \boldsymbol{\sigma} \\
& +\operatorname{div}\left\{\boldsymbol{H}^{\mathrm{Re}}\left(\boldsymbol{E}^{\mathrm{Im}} \cdot \boldsymbol{\sigma}\right)-\boldsymbol{H}^{\mathrm{Im}}\left(\boldsymbol{E}^{\mathrm{Re}} \cdot \boldsymbol{\sigma}\right)+\boldsymbol{E}^{\mathrm{Im}}\left(\boldsymbol{H}^{\mathrm{Re}} \cdot \boldsymbol{\sigma}\right)\right. \\
& \left.-\boldsymbol{E}^{\operatorname{Re}}\left(\boldsymbol{H}^{\mathrm{Im}} \cdot \boldsymbol{\sigma}\right)-\left[\left(\boldsymbol{E}^{\mathrm{Im}} \cdot \boldsymbol{H}^{\mathrm{Re}}\right)-\left(\boldsymbol{E}^{\mathrm{Re}} \cdot \boldsymbol{H}^{\mathrm{Im}}\right)\right] \boldsymbol{\sigma}\right\}=0 .
\end{aligned}
$$

[1] Da Silveira, Z. Naturforsch. 34a, 646 (1979).

[2] H. Sallhofer, Z. Naturforsch. 33 a, 1378 (1978).
The first two terms in curly brackets vanish for any charge-free electrodynamics according to $[3,(1)]$. Using $[4,(5)]$ and $[4,(6)]$ the remainder can be put into the form

$$
(\dot{\boldsymbol{V}}=\operatorname{div} \mathbf{T}) \cdot \boldsymbol{\sigma}=0,
$$

or more generally

$$
\dot{\boldsymbol{V}}+\operatorname{div} \mathbf{T}=0
$$

in agreement with [4, (11)].

The above derivation of the momentum balance suggests, among others, that the structure of Diraclike electrodynamics is closely related to the Pauli matrices.

[3] H. Sallhofer, Z. Naturforsch. 34a, 1145 (1979).

[4] H. Sallhofer, Z. Naturforsch. 39a, 1142 (1984). 